\title{
On the boundary conditions for EG-methods applied to the two-dimensional wave equation system
}

\author{
M. Lukáčová-Medviđová ${ }^{1}{ }^{2}$, G. Warnecke $^{1}$ and Y. Zahaykah ${ }^{1}$
}

\begin{abstract}
The subject of the paper is the study of some nonreflecting and reflecting boundary conditions for the evolution Galerkin methods (EG) which are applied for the two-dimensional wave equation system. Different known tools are used to achieve this aim. Namely, the method of characteristics, the method of extrapolation, the Laplace transformation method, and the perfectly matched layer (PML) method. We show that the absorbing boundary conditions which are based on the use of the Laplace transformation lead to the Engquist-Majda first and second order absorbing boundary conditions, see [3]. Further, following Bérenger [1] we consider the PML method. We discretize the wave equation system with the leap-frog scheme inside the PML while the evolution Galerkin schemes are used inside the computational domain. Numerical tests demonstrate that this method produces much less unphysical reflected waves as well as the best results in comparison with other techniques studied in the paper.
\end{abstract}

Key words: hyperbolic systems, wave equation, evolution Galerkin schemes, absorbing boundary conditions, reflecting boundary conditions, perfectly matched layer, Laplace transformation.

\section{Introduction}

It is well known that to solve a differential equation numerically in an unbounded domain, it is necessary to restrict the computations to a bounded domain $\Omega$. Therefore we need to introduce boundary conditions on artificial boundaries. As a consequence the question arises "is there a boundary condition such that the solution of the problem in $\Omega$ together with this boundary condition coincides exactly with the restriction to $\Omega$ of the solution in the unbounded domain". Such boundary conditions are called nonreflecting boundary conditions or absorbing boundary conditions.

On the other hand in the case of a physical boundary it is necessary to reflect the solution (wave) from the boundary completely. These boundary conditions are called reflecting boundary conditions.

Regarding the above question, many techniques were developed either for the scalar wave equation or for linear systems of hyperbolic equations, see, e.g. Grote and Keller [5, 6, 7], Engquist and Majda [3], Higdon [9], Thompson [19, 20] and Bradly, Greengard and Hagstrom [2]. Moreover such absorbing boundary conditions appear often in electromagnetic computations where many such conditions were developed, see e.g. the matched layer [10] which is based

\footnotetext{
${ }^{1}$ Institut für Analysis und Numerik, Otto-von-Guericke-Universität Magdeburg, Universitätsplatz 2, 39106 Magdeburg, Germany, emails: Gerald.Warnecke@mathematik.uni-magdeburg.de Yousef.Zahaykah@mathematik.uni-magdeburg.de

${ }^{2} \mathrm{TU}$ Hamburg-Harburg, Arbeitsbereich Mathematik, Schwarzenbergstrasse 95, 21073 Hamburg email:lukacova@tu-harburg.de
} 
on the idea of surrounding the computational domain with an absorbing medium whose impedance matches that of the free space. Others are the Mur conditions [16], a perfectly matched layer for the absorption of electromagnetic waves [1] and many others.

In this paper known nonreflecting and reflecting boundary conditions for the two dimensional wave equation system are considered and applied for the evolution Galekin methods (EG). The EG methods were studied by Lukáčová, Morton and Warnecke in [12], [13] for the multidimensional hyperbolic systems. These methods belong to the class of genuinely multidimensional schemes. Typically the dimensional splitting FV methods approximate solution only in normal directions to cell interfaces. On the other hand the EG methods use the approximate evolution Galerkin operators, which are based on the use of the bicharacteristics of multi-dimensional hyperbolic systems, such that all of the infinitely many directions of the wave propagation are taken into account, see Section 2. Until now, EG methods had been applied to easy test cases with periodic or other simple boundary data. For realy interesting physical applications it is necessary to demonstrate that the EG schemes are compatible with more sophisticated numerical boundary treatments.

The outline of the paper will be as follows: in the next section we give a brief description of EG methods. For more details on these schemes the reader is referred to [12], [13], [14], [15], [17]. In the second section we follow Thompson [19], [20] and use the method of characteristics to derive nonreflecting boundary conditions as well as reflecting boundary conditions for the wave equation system. We also apply directly extrapolation to the wave equation system to produce an absorbing boundary condition. This does not always produce satisfactory numerical results, see e.g. [14]. Therefore we consider other numerical techniques. Moreover in Section 3 we link the Laplace transformation and Fourier series to the two-dimensional wave equation system and derive Engquist-Majda, [3], absorbing boundary conditions. In the last part of Section 3 we briefly consider the absorbing boundary conditions based on a perfectly matched layer (PML) which develped by Berenger [1]. In the third section we present results of numerical experiments. The above boundary conditions will be combined with the EG methods inside the computational domain. We use particularly the EG3, EG4 first order schemes and the FVEG3 second order scheme, see Section 2. Finally, we emphasize that the best results were obtained when the PML is used.

\section{Evolution Galerkin methods}

Evolution Galerkin methods, EG-methods, were proposed to approximate hyperbolic conservation laws. An important feature of such methods is that they take into account better the infinitely many directions of propagation of waves in multidimensional cases. It is well-known, see [12], [13], [14], [15], [17], that a basic tool to derive these schemes is the general theory of bicharacteristics of linear hyperbolic systems. This theory is used to derive the system of integral equations which is equivalent to the concerned first order system, e.g. the wave equation system. Using certain types of quadratures, these integral equations lead to the approximate evolution operator that build up the evolution Galerkin scheme.

Let the general form of linear hyperbolic system be given as

$$
\mathbf{U}_{t}+\sum_{j=1}^{d} \mathbf{A}_{j} \mathbf{U}_{x_{j}}=0, \mathbf{x}=\left(x_{1}, \ldots, x_{d}\right)^{T} \in \mathbb{R}^{d}
$$




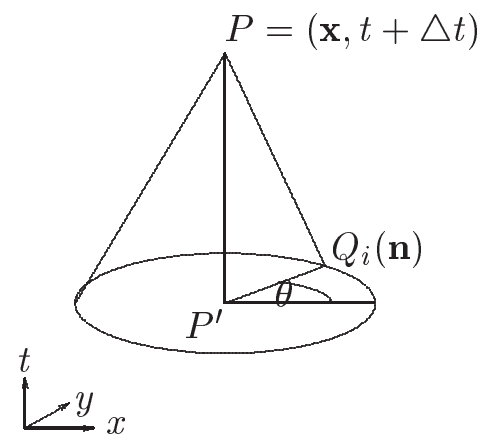

Figure 1: Bicharacteristics along the Mach cone through $P$ and $Q_{i}(\mathbf{n})$

where the coefficient matrices $\mathbf{A}_{j}, j=1, \ldots, d$ are elements of $\mathbb{R}^{p \times p}$ and the dependent variables are $\mathbf{U}=\left(u_{1}, \ldots, u_{p}\right)^{T} \in \mathbb{R}^{p}$. Let $\mathbf{A}(\mathbf{n})=\sum_{j=1}^{d} n_{j} \mathbf{A}_{j}$ be the pencil matrix with $\mathbf{n}=$ $\left(n_{1}, \ldots, n_{d}\right)^{T}$ being a directional vector in $\mathbb{R}^{d}$. Then using the eigenvectors of $\mathbf{A}(\mathbf{n})$ system (2.1) can be rewritten in a characteristic form via the substitution $\mathbf{W}=\mathbf{R}^{-1} \mathbf{U}$, where the columns of the matrix $\mathbf{R}$ are the linearly independent right eigenvectors of $\mathbf{A}(\mathbf{n})$. Since the coefficients of the original system are constants, the bicharacteristics of the resulting characteristic system are straight lines $P Q_{i}$ and $P P^{\prime}$ generating the mantle of the Mach cone, see Figure 1. Diagonalizing this system and integrating along the bicharacteristics lead to the following system of integral equations

$$
\mathbf{U}(P)=\frac{1}{|O|} \int_{O} \mathbf{R}(\mathbf{n})\left[\begin{array}{c}
W_{1}\left(Q_{1}(\mathbf{n}), \mathbf{n}\right) \\
\vdots \\
W_{p}\left(Q_{p}(\mathbf{n}), \mathbf{n}\right)
\end{array}\right] \mathrm{d} O+\frac{1}{|O|} \int_{O} \int_{0}^{\Delta t} \mathbf{R}(\mathbf{n}) \mathbf{S}(t+\tau, \mathbf{n}) \mathrm{d} \tau \mathrm{d} O .
$$

Where $O$ is the unit sphere in $\mathbb{R}^{d},|O|$ its surface measure and $S$ is a nontrivial term which we call the source term. For more details see [13].

Next we give the approximate evolution operators for the two-dimensional wave equation system (3.1) of two EG schemes, namely the EG3 and the EG4 schemes.

\section{Approximate evolution operators:}

- EG3 scheme:

$$
\begin{aligned}
\phi_{P}=\frac{1}{2 \pi} \int_{0}^{2 \pi}\left(\phi_{Q}-2 u_{Q} \cos \theta-2 v_{Q} \sin \theta\right) \mathrm{d} \theta+O\left(\Delta t^{2}\right), \\
u_{P}=\frac{1}{2} u_{P^{\prime}}+\frac{1}{2 \pi} \int_{0}^{2 \pi} \begin{array}{l}
\left(-2 \phi_{Q} \cos \theta+u_{Q}\left(3 \cos ^{2} \theta-1\right)+3 v_{Q} \sin \theta \cos \theta\right) \mathrm{d} \theta \\
+
\end{array} \\
v_{P}=\frac{1}{2} v_{P^{\prime}}+\frac{1}{2 \pi} \int_{0}^{2 \pi} \begin{array}{c}
\left(-2 t_{Q} \sin \theta+3 u_{Q} \sin \theta \cos \theta+v_{Q}\left(3 \sin ^{2} \theta-1\right)\right) \mathrm{d} \theta \\
+
\end{array} \\
+O\left(\Delta t^{2}\right) .
\end{aligned}
$$


- EG4 scheme:

$$
\begin{aligned}
& \phi_{P}=\frac{1}{2 \pi} \int_{0}^{2 \pi}\left(\phi_{Q}-2 u_{Q} \cos \theta-2 v_{Q} \sin \theta\right) \mathrm{d} \theta+O\left(\Delta t^{2}\right) \\
& u_{P}=\frac{1}{2 \pi} \int_{0}^{2 \pi}\left(-2 \phi_{Q} \cos \theta+2 u_{Q} \cos ^{2} \theta+2 v_{Q} \sin \theta \cos \theta\right) \mathrm{d} \theta+O\left(\Delta t^{2}\right), \\
& v_{P}=\frac{1}{2 \pi} \int_{0}^{2 \pi}\left(-2 \phi_{Q} \sin \theta+2 u_{Q} \sin \theta \cos \theta+2 v_{Q} \sin ^{2} \theta\right) \mathrm{d} \theta+O\left(\Delta t^{2}\right)
\end{aligned}
$$

\section{Evoution Galerkin schemes:}

For simplicity let us take $d=2$. Consider $h>0$ to be the mesh size parameter. We construct a mesh for $\mathbb{R}^{2}$, which consists of the square mesh cells

$$
\Omega_{k l}=\left[\left(k-\frac{1}{2}\right) h,\left(k+\frac{1}{2}\right) h\right] \times\left[\left(l-\frac{1}{2}\right) h,\left(l+\frac{1}{2}\right) h\right]=\left[x_{k}-\frac{h}{2}, x_{k}+\frac{h}{2}\right] \times\left[y_{l}-\frac{h}{2}, y_{l}+\frac{h}{2}\right],
$$

where $k, l \in \mathbb{Z}$. Let us denote by $H^{\kappa}\left(\mathbb{R}^{2}\right)$ the Sobolev space of distributions with derivatives up to order $\kappa$ in $L^{2}$ space, where $\kappa \in \mathbb{N}$. Consider the general hyperbolic system given by the equation (2.1). Let us denote by $E(s):\left(H^{\kappa}\left(\mathbb{R}^{2}\right)\right)^{p} \rightarrow\left(H^{\kappa}\left(\mathbb{R}^{2}\right)\right)^{p}$ the exact evolution operator for the system (2.1), i.e.

$$
\mathbf{U}(., t+s)=E(s) \mathbf{U}(., t) .
$$

We suppose that $S_{h}^{m}$ is a finite element space consisting of piecewise polynomials of order $m \geq 0$ with respect to the square mesh. Assume constant time step, i.e. $t_{n}=n \Delta t$. Let $\mathbf{U}^{n}$ be an approximation in the space $S_{h}^{m}$ to the exact solution $\mathbf{U}\left(., t_{n}\right)$ at time $t_{n} \geq 0$. We consider $E_{\tau}: L_{l o c}^{1}\left(\mathbb{R}^{2}\right) \rightarrow\left(H^{\kappa}\left(\mathbb{R}^{2}\right)\right)^{p}$ to be a suitable approximate evolution operator for $E(\tau)$. In practice we will use restrictions of $E_{\tau}$ to the subspace $S_{h}^{m}$ for $m \geq 0$. Then we can define the general class of evolution Galerkin methods.

Definition 2.5 Starting from some initial data $\mathbf{U}^{0} \in S_{h}^{m}$ at time $t=0$, an evolution Galerkin method (EG-method) is recursively defined by means of

$$
\mathbf{U}^{n+1}=P_{h} E_{\tau} \mathbf{U}^{n},
$$

where $P_{h}$ is the $L^{2}$-projection given by the integral averages in the following way

$$
\left.P_{h} \mathbf{U}^{n}\right|_{\Omega_{k l}}=\frac{1}{\left|\Omega_{k l}\right|} \int_{\Omega_{k l}} \mathbf{U}\left(x, y, t_{n}\right) d x d y .
$$

We denote by $R_{h}: S_{h}^{m} \rightarrow S_{h}^{r}$ a recovery operator, $r \geq m \geq 0$ and consider our approximate evolution operator $E_{\tau}$ on $S_{h}^{r}$. We will limit our further considerations to the case where $m=0$ and $r=1$. Taking piecewise constants the resulting schemes will only be of first order, even when $E_{\tau}$ is approximated to a higher order. Higher order accuracy can be obtained either by taking $m>0$, or by inserting a recovery stage $R_{h}$ before the evolution step in equation $(2.6)$ to give

$$
\mathbf{U}^{n+1}=P_{h} E_{\tau} R_{h} \mathbf{U}^{n} .
$$

This approach involves the computation of multiple integrals and becomes quite complex for higher order recoveries. To avoid this we will consider higher order evolution Galerkin schemes based on the finite volume formulation instead. 
Definition 2.8 Starting from some initial data $\mathbf{U}^{0} \in S_{h}^{m}$, the finite volume evolution Galerkin method (FVEG) is recursively defined by means of

$$
\mathbf{U}^{n+1}=\mathbf{U}^{n}-\frac{1}{h} \int_{0}^{\Delta t} \sum_{j=1}^{2} \delta_{x_{j}} \mathbf{f}_{j}\left(\tilde{\mathbf{U}}^{n+\frac{\tau}{\Delta t}}\right) d \tau,
$$

where $\delta_{x_{j}} \mathbf{f}_{j}\left(\tilde{\mathbf{U}}^{n+\frac{\tau}{\Delta t}}\right)$ represents an approximation to the edge flux difference and $\delta_{x}$ is defined by $\delta_{x}=v\left(x+\frac{h}{2}\right)-v\left(x-\frac{h}{2}\right)$. The cell boundary value $\tilde{\mathbf{U}}^{n+\frac{\tau}{\Delta t}}$ is evolved using the approximate evolution operator $E_{\tau}$ to $t_{n}+\tau$ and averaged a long the cell boundary, i.e.

$$
\tilde{\mathbf{U}}^{n+\frac{\tau}{\Delta t}}=\sum_{k, l \in \mathbb{Z}}\left(\frac{1}{\left|\partial \Omega_{k l}\right|} \int_{\partial \Omega_{k l}} E_{\tau} R_{h} \mathbf{U}^{n} d S\right) \chi_{k l},
$$

where $\chi_{k l}$ is the characteristic function of $\partial \Omega_{k l}$.

In this formulation a first order approximation $E_{\tau}$ to the exact operator $E(\tau)$ yields an overall higher order update from $\mathbf{U}^{n}$ to $\mathbf{U}^{n+1}$. To obtain this approximation in the discrete scheme it is only necessary to carry out a recovery stage at each level to generate a piecewise polynomial approximation $\tilde{\mathbf{U}}^{n}=R_{h} \mathbf{U}^{n} \in S_{h}^{r}$ from the piecewise constant $\mathbf{U}^{n} \in S_{h}^{0}$, to feed into the calculation of the fluxes. To construct the second order FVEG schemes, for example, we take the first order accurate approximate evolution operator and define a bilinear reconstruction $R_{h}$. Among many possible recovery schemes, which can be used, we will choose a discontinuous bilinear recovery using four point averages at each vertex. It is given as

$$
\begin{aligned}
\left.R_{h} \mathbf{U}\right|_{\Omega_{k l}} & =\mathbf{U}_{k l}+\frac{\left(x-x_{k}\right)}{4 h}\left(\Delta_{0 x} \mathbf{U}_{k l+1}+2 \Delta_{0 x} \mathbf{U}_{k l}+\Delta_{0 x} \mathbf{U}_{k l-1}\right) \\
& +\frac{\left(y-y_{l}\right)}{4 h}\left(\Delta_{0 y} \mathbf{U}_{k+1 l}+2 \Delta_{0 y} \mathbf{U}_{k l}+\Delta_{0 y} \mathbf{U}_{k-1 l}\right) \\
& +\frac{\left(x-x_{k}\right)\left(y-y_{l}\right)}{h^{2}} \Delta_{0 y} \Delta_{0 x} \mathbf{U}_{k l},
\end{aligned}
$$

where $\Delta_{0 z} v(z)=\frac{1}{2}(v(z+h)-v(z-h))$. Note that in the updating step (2.9) some numerical quadratures are used instead of the exact time integration. Similarly, to evaluate the intermediate value $\tilde{\mathbf{U}}^{n+\frac{\tau}{\Delta t}}$ in (2.10) either the two dimensional integrals along the cellinterface and around the Mach cone are evaluated exactly or by means of suitable numerical quadratures. Since the approximate evolution operator $E_{\tau}$ contains integral a long the base cone (circle from 0 to $2 \pi$ ), see Figure 1 and equations (2.2) and (2.3), it is difficult to involve boundary conditions in Definitions 2.5 and 2.8. In the next section we will use well known techniques to derive suitable boundary conditions to be used with EG-methods.

\section{Boundary conditions}

Consider the Cauchy problem for $c>0$ and $(x, y) \in \mathbb{R}^{2}$

$$
\begin{aligned}
& \frac{\partial \phi}{\partial t}-c \nabla \cdot\left(\begin{array}{c}
u \\
v
\end{array}\right)=0 \\
& \frac{\partial}{\partial t}\left(\begin{array}{l}
u \\
v
\end{array}\right)-c \nabla \phi=0
\end{aligned}
$$




$$
\begin{aligned}
& \phi(x, y, 0)=\phi_{0}(x, y), \\
& u(x, y, 0)=u_{0}(x, y), \\
& v(x, y, 0)=v_{0}(x, y) .
\end{aligned}
$$

Let us take the computational domain to be $\Omega=[0, \alpha] \times[0, \beta] \subset \mathbb{R}^{2}$. Moreover, assume that there is no physical boundary, i.e. $\Omega$ is obtained only by truncation of the whole domain $\mathbb{R}^{2}$. As we mentioned, to derive appropriate boundary conditions for this system we will consider known techniques as follows:

\subsection{Boundary conditions based on characteristic methods}

In this subsection we use the method of characteristics, Thompson [19], [20], and derive artificial boundary conditions at the artificial interfaces $x=0, x=\alpha, y=0$, and $y=\beta$ of the computational domain. Now system (3.1) can be rewritten in the matrix form

$$
\mathbf{U}_{t}+\mathbf{A}_{1} \mathbf{U}_{x}+\mathbf{A}_{2} \mathbf{U}_{y}=0,
$$

where

$$
\mathbf{U}:=\left(\begin{array}{c}
\phi \\
u \\
v
\end{array}\right), \quad \mathbf{A}_{1}:=\left(\begin{array}{rrr}
0 & -c & 0 \\
-c & 0 & 0 \\
0 & 0 & 0
\end{array}\right), \quad \mathbf{A}_{2}:=\left(\begin{array}{rrr}
0 & 0 & -c \\
0 & 0 & 0 \\
-c & 0 & 0
\end{array}\right) .
$$

Put $\mathbf{C}=\mathbf{A}_{2} \mathbf{U}_{y}$ and denote the matrix of the right eigenvectors of the coefficient matrix $\mathbf{A}_{1}$ by $\mathbf{S}=\left[\mathbf{r}_{1}\left|\mathbf{r}_{2}\right| \mathbf{r}_{3}\right]$. It holds

$$
\mathbf{S}^{-1} \mathbf{A}_{1} \mathbf{S}=\boldsymbol{\Lambda}_{1}, \quad \text { where } \Lambda_{1 i j}=\left\{\begin{aligned}
0 & \text { if } i \neq j, \\
\lambda_{i} & \text { if } i=j, i, j=1,2,3 .
\end{aligned}\right.
$$

With these notations system (3.3) can be written as

$$
\mathbf{S}^{-1} \frac{\partial \mathbf{U}}{\partial t}+\boldsymbol{\Lambda}_{1} \mathbf{S}^{-1} \frac{\partial \mathbf{U}}{\partial x}+\mathbf{S}^{-1} \mathbf{C}=0
$$

or we can write it in the form

$$
\mathbf{1}_{i}^{T} \frac{\partial \mathbf{U}}{\partial t}+\lambda_{i} \mathbf{1}_{i}^{T} \frac{\partial \mathbf{U}}{\partial x}+\mathbf{l}_{i}^{T} \mathbf{C}=0
$$

where $\mathbf{l}_{i}^{T}$ is a left eigenvector of $\mathbf{A}_{1}$ and $\lambda_{i}$ is the corresponding eigenvalue. Define $L_{i}$ to be

$$
L_{i}:=\lambda_{i} T_{i}^{T} \frac{\partial \mathbf{U}}{\partial x}
$$

then equation (3.4) has the form

$$
\mathbf{l}_{i}^{T} \frac{\partial \mathbf{U}}{\partial t}+L_{i}+\mathbf{l}_{i}^{T} \mathbf{C}=0
$$

or equivalently

$$
\frac{\partial \mathbf{U}}{\partial t}+\mathbf{S} \mathbf{L}+\mathbf{C}=0
$$

where $\mathbf{L}:=\left(L_{1}, L_{2}, L_{3}\right)^{T}$. An easy calculation shows that the eigenvalues of the matrix $\mathbf{A}_{1}$ are $-c, 0, c$, and the corresponding left and right eigenvectors are

$$
\mathbf{l}_{1}^{T}=\left(\frac{1}{\sqrt{2}}, \frac{1}{\sqrt{2}}, 0\right), \mathbf{l}_{2}^{T}=(0,0,1), \mathbf{l}_{3}^{T}=\left(-\frac{1}{\sqrt{2}}, \frac{1}{\sqrt{2}}, 0\right)
$$




$$
\mathbf{r}_{1}=\left(\begin{array}{c}
\frac{1}{\sqrt{2}} \\
\frac{1}{\sqrt{2}} \\
0
\end{array}\right), \mathbf{r}_{2}=\left(\begin{array}{l}
0 \\
0 \\
1
\end{array}\right), \mathbf{r}_{3}=\left(\begin{array}{r}
-\frac{1}{\sqrt{2}} \\
\frac{1}{\sqrt{2}} \\
0
\end{array}\right),
$$

respectively. Using the definition of $L_{i}$, equation (3.5), and the above left eigenvectors we get

$$
\begin{aligned}
L_{1} & :=\lambda_{1} \mathbf{l}_{1}^{T} \frac{\partial \mathbf{U}}{\partial x}=\frac{-c}{\sqrt{2}}\left(\frac{\partial \phi}{\partial x}+\frac{\partial u}{\partial x}\right), \\
L_{2} & :=\lambda_{2} \mathbf{l}_{2}^{T} \frac{\partial \mathbf{U}}{\partial x}=0, \\
L_{3} & :=\lambda_{3} \mathbf{l}_{3}^{T} \frac{\partial \mathbf{U}}{\partial x}=\frac{c}{\sqrt{2}}\left(\frac{-\partial \phi}{\partial x}+\frac{\partial u}{\partial x}\right) .
\end{aligned}
$$

Moreover we have

$$
\mathbf{S} \mathbf{L}=\left(\begin{array}{ccc}
\frac{1}{\sqrt{2}} & 0 & \frac{-1}{\sqrt{2}} \\
\frac{1}{\sqrt{2}} & 0 & \frac{1}{\sqrt{2}} \\
0 & 1 & 0
\end{array}\right)\left(\begin{array}{c}
L_{1} \\
L_{2} \\
L_{3}
\end{array}\right)=\left(\begin{array}{c}
\frac{1}{\sqrt{2}}\left(L_{1}-L_{3}\right) \\
\frac{1}{\sqrt{2}}\left(L_{1}+L_{3}\right) \\
L_{2}
\end{array}\right) .
$$

Now substituting from (3.8) into (3.6) leads to the system

$$
\begin{aligned}
\frac{\partial \phi}{\partial t}+\frac{1}{\sqrt{2}}\left(L_{1}-L_{3}\right)-c \frac{\partial v}{\partial y} & =0, \\
\frac{\partial u}{\partial t}+\frac{1}{\sqrt{2}}\left(L_{1}+L_{3}\right) & =0, \\
\frac{\partial v}{\partial t}+L_{2}-c \frac{\partial \phi}{\partial y} & =0 .
\end{aligned}
$$

This system is used for the update of the solution on the interfaces $x=0$ and $x=\alpha$, while in the interior domain $\{(x, y):(x, y) \in] 0, \alpha[\times] 0, \beta[\}$ we solve system (3.1) together with the initial data (3.2). Now the transverse derivatives, i.e. the $y$ derivatives, in (3.9) and (3.11) are evaluated as usual. The values of the $L_{i}$ are determined depending on the sign of the corresponding eigenvalues $\lambda_{i}$ at the corresponding interface. Namely, at the interface $x=0$ the eigenvalues are $-c, 0, c$, which from (3.7) immediately gives that $L_{2}$ must be zero. The wave corresponding to the eigenvalue $\lambda_{1}=-c<0$ is an outgoing one that leaves the computational domain. Thus $L_{1}$ is evaluated directly from its definition (3.7). Since $l_{3}$ is matched with the positive eigenvalue $\lambda_{3}=c$ the corresponding wave is an incoming one. It is this lack of information that prevents us from using the definition to evaluate $L_{3}$. Let us drop the transverse derivative from equation (3.9), then subtracting (3.9) from (3.10) we end with the relation

$$
\frac{\partial(-\phi+u)}{\partial t}+\frac{2}{\sqrt{2}} L_{3}=0 .
$$

Now $-\phi+u$ is the one-dimensional characteristic wave that enters the computational domain. Preventing this wave from entering the computational domain is equivalent to taking its time derivative equal to zero, see [8]. This forces $L_{3}$ to be equal to zero. A similar argument for the interface $x=\alpha$ implies that $L_{1}$ and $L_{2}$ must be zero while $L_{3}$ is evaluated from its definition (3.7).

For the case $y=0$ and $y=\beta$ we use a similar argument as above. At corners we combine both 
cases. It is important to note that the method of characteristics can be used to construct not only absorbing boundary conditions but also reflecting ones. To show this, assume that the interface $x=0$ is a reflector. To define a reflected boundary condition at $x=0$ the function $\phi$ must vanish for all time $t>0$ at $x=0$. Then equation (3.9) implies that $L_{3}=L_{1}-\sqrt{2} c \frac{\partial v}{\partial y}$.

Remark 3.12 One of the simplest absorbing boundary conditions is to extrapolate the data from the interior domain to the cells that lie on the boundary. Mathematically, for the wave equation system (3.1), this can be done by taking $\frac{\partial \varphi}{\partial s}=0$, where $\varphi \in\{\phi, u, v\}$ and the derivative with respect to $s$ stands for the directional derivative in some direction parametrized by the parameter s. We will show however in our numerical experiments that this approach can lead to considerable unphysical reflections in the solution.

\subsection{Absorbing boundary conditions based on the Laplace transformation}

In this subsection we recall the use of Fourier series and the Laplace transformation to construct an absorbing boundary condition to be used, say, at the boundary $\{(\alpha, y): 0 \leq y \leq \beta\}$. We will expand the solution of the wave equation system in a complex Fourier series. Substituting into the wave equation system and applying the Laplace transformation allows a derivation of Engquist-Majda [3] absorbing boundary conditions.

Consider the wave equation system (3.1) together with the initial condition (3.2) in $\mathbb{R}^{2}$. Suppose that the initial functions $\phi_{0}(x, y), u_{0}(x, y)$ and $v_{0}(x, y)$ are all smooth and vanish outside the domain $\Omega=[0, \alpha] \times[0, \beta]$. We look for a boundary condition that holds at the boundary of the domain $\partial \Omega$ such that the solution of system (3.1) accompanied with the initial data (3.2) on the domain $\Omega$ together with this condition coincides with the solution on the unbounded domain. The Laplace transformation of a function $f(t)$ will be denoted by $\hat{f}(s)=L(f(t))$, and its inverse by $f(t)=L^{-1}(\hat{f}(s))$. They are defined as follows

$$
\begin{gathered}
\hat{f}(s):=L(f(t))=\int_{0}^{\infty} f(t) \exp (-s t) \mathrm{d} t \\
f(t):=L^{-1}(\hat{f}(s))=\frac{1}{2 \pi i} \int_{a-i \infty}^{a+i \infty} \hat{f}(s) \exp (s t) \mathrm{d} t .
\end{gathered}
$$

To produce an exact absorbing boundary condition using the Laplace transformation we use the Fourier series and expand the solution $\mathbf{U}(x, y, t)$ at the points to the right of the line $x=\alpha$ as

$$
\mathbf{U}(x, y, t)=\sum_{k=-\infty}^{\infty} \mathbf{U}^{k}(x, t) \exp i\left(k \frac{\pi}{\beta} y\right)
$$

Substituting (3.12) into the wave equation system (3.3) yields

$$
\mathbf{U}_{t}^{k}+\mathbf{A}_{1} \mathbf{U}_{x}^{k}+i k \frac{\pi}{\beta} \mathbf{A}_{2} \mathbf{U}^{k}=0
$$

Applying the Laplace transformation to (3.13) and using the properties $L\left(f^{\prime}(t)\right)=s L(f(t))$ $f^{\prime}(0)$ and $L\left(\frac{\partial f}{\partial x}\right)=\frac{\partial}{\partial x}(L(f))$ as well as the assumption that the initial data vanish in the exterior of $\Omega$, we get

$$
s_{s} \hat{\mathbf{U}}^{k}(x, s)+\mathbf{A}_{1} \hat{\mathbf{U}}_{x}^{k}(x, s)+i k \frac{\pi}{\beta} \mathbf{A}_{2} \hat{\mathbf{U}}^{k}(x, s)=0 .
$$


Expressing $\hat{u}^{k}$ and $\hat{v}^{k}$ in terms of $\hat{\phi}^{k}$ we end with the following second order differential equation

$$
\hat{\phi}_{x x}^{k}-\left[\frac{s^{2}}{c^{2}}-\left(\frac{i k \pi}{\beta}\right)^{2}\right] \hat{\phi}^{k}=0 .
$$

The boundedness of the solution for $x \rightarrow \infty$ implies that the solution of (3.15) is

$$
\hat{\phi}^{k}(x, s)=A(s, y) \exp \left(-\frac{s}{c} \sqrt{1-\left(\frac{i c k \pi}{s \beta}\right)^{2}} x\right) .
$$

The values of $\hat{u}^{k}$ and $\hat{v}^{k}$ read

$$
\begin{aligned}
& \hat{u}^{k}(x, s)=-\sqrt{1-\left(\frac{i c k \pi}{s \beta}\right)^{2}} A(s, y) \exp \left(-\frac{s}{c} \sqrt{1-\left(\frac{i c k \pi}{s \beta}\right)^{2}} x\right), \\
& \hat{v}^{k}(x, s)=\frac{i c k \pi}{s \beta} A(s, y) \exp \left(-\frac{s}{c} \sqrt{1-\left(\frac{i c k \pi}{s \beta}\right)^{2}} x\right) .
\end{aligned}
$$

If we apply the operator

$$
\mathcal{L}=\left(\frac{\partial}{\partial x}+\frac{s}{c} \sqrt{1-\left(\frac{i c k \pi}{s \beta}\right)^{2}}\right)
$$

to the functions $\hat{\phi}^{k}, \hat{u}^{k}$ and $\hat{v}^{k}$ we get $\mathcal{L} \hat{\phi}^{k}=0, \mathcal{L} \hat{u}^{k}=0$ and $\mathcal{L} \hat{v}^{k}=0$. Hence, at $x=\alpha$, we have the following boundary condition for each component $\mathbf{u}^{k}(x, t)$

$$
L^{-1}\left\{\mathcal{L} \hat{\phi}^{k}\right\}=0, L^{-1}\left\{\mathcal{L} \hat{u}^{k}\right\}=0, L^{-1}\left\{\mathcal{L} \hat{v}^{k}\right\}=0 .
$$

Let $g(t)=L^{-1}\left(s \sqrt{1-\frac{a^{2}}{s^{2}}}\right)$, where $a=i c k \frac{\pi}{\beta}$, then at $x=\alpha$ condition (3.20) can be rewritten as

$$
\begin{aligned}
\phi_{x}^{k}(x, t)+\frac{1}{c} \int_{0}^{t} g(t-\tau) \phi^{k}(x, \tau) \mathrm{d} \tau & =0 \\
u_{x}^{k}(x, t)+\frac{1}{c} \int_{0}^{t} g(t-\tau) u^{k}(x, \tau) \mathrm{d} \tau & =0 \\
v_{x}^{k}(x, t)+\frac{1}{c} \int_{0}^{t} g(t-\tau) v^{k}(x, \tau) \mathrm{d} \tau & =0 .
\end{aligned}
$$

It is clear from equations (3.21) that this boundary condition is local in position but unfortunately not local in time. Using $\sqrt{1-\frac{a^{2}}{x^{2}}}=1+O\left(\frac{1}{x^{2}}\right)$ we approximate the operator $\mathcal{L}$ as $\mathcal{L}=\frac{\partial}{\partial x}+\frac{s}{c}$. Hence (3.20) gives the following local in time Engquist-Majda first order absorbing boundary condition for the function $\phi$ at the interface $x=\alpha$.

$$
\frac{\partial \phi}{\partial x}+\frac{1}{c} \frac{\partial \phi}{\partial t}=0
$$


Using $\sqrt{1-\frac{a^{2}}{x^{2}}}=1-\frac{a^{2}}{2 x^{2}}+O\left(\frac{1}{x^{4}}\right)$ we approximate the operator $\mathcal{L}$ as $\mathcal{L}=\frac{\partial}{\partial x}+\frac{s}{c}\left(1-\frac{a^{2}}{2 s^{2}}\right)$. If we substitute $s \mathcal{L}$ into (3.20) then we obtain the following Engquist-Majda second order absorbing boundary condition for $\phi$ at $x=\alpha$.

$$
\frac{\partial^{2} \phi}{\partial x \partial t}+\frac{1}{c} \frac{\partial^{2} \phi}{\partial t^{2}}-\frac{c}{2} \frac{\partial^{2} \phi}{\partial y^{2}}=0
$$

Note that the absorbing boundary conditions for other interfaces can be derived in an analogous way. For the descritization of (3.22) and (3.23) we refere to [3].

\subsection{Absorbing boundary condition based on a perfectly matched layer}

In the previous two subsections absorbing boundary conditions were defined using certain types of operators. Although such boundary conditions absorb the incoming waves, the absorption is not complete for some cases, as we will see in the numerical examples in the next section. By construction, such boundary conditions are derived to absorb the reflected waves provided that the incident plane waves are normal to the interfaces, for more details see Grote [4]. In this subsection we will apply boundary conditions that are valid independently of the direction of the incident wave or its frequency. This type of boundary conditions was introduced by Bérenger [1] for electromagnetic problems. Afterwards many authors have been using such technique, for example, Fang [11] and Quan and Geers [18]. The main point is to surround the computational domain by a layer that has the same impedance, roughly speaking resistance to the wave propagation, as the free domain, the domain where the wave equation system (3.1) valid. Thus for plane waves, at least theoretically, Berenger has shown, for the electromagnetic case, that there is no reflection. For more details on PML method see [1], [11], [18]. To define PML layers we split the function $\phi$ into two components $\phi^{x}$ and $\phi^{y}$, i.e. we write $\phi=\phi^{x}+\phi^{y}$. Then we define

Definition 3.24 A layer $\Omega\left(\subset \mathbb{R}^{2}\right)$ in which the system, damped wave equation system,

$$
\begin{aligned}
& \frac{\partial}{\partial t}\left(\begin{array}{c}
\phi^{x} \\
\phi^{y}
\end{array}\right)+\left(\begin{array}{cc}
\sigma_{x}^{\star} & 0 \\
0 & \sigma_{y}^{\star}
\end{array}\right)\left(\begin{array}{c}
\phi^{x} \\
\phi^{y}
\end{array}\right)-c\left(\begin{array}{c}
\frac{\partial u}{\partial x} \\
\frac{\partial v}{\partial y}
\end{array}\right)=0, \\
& \frac{\partial}{\partial t}\left(\begin{array}{c}
u \\
v
\end{array}\right)+\left(\begin{array}{cc}
\sigma_{x} & 0 \\
0 & \sigma_{y}
\end{array}\right)\left(\begin{array}{l}
u \\
v
\end{array}\right)-c \nabla\left(\phi^{x}+\phi^{y}\right)=0 .
\end{aligned}
$$

is satisfied where $\sigma_{x}, \sigma_{x}^{\star}, \sigma_{y}$, and $\sigma_{y}^{\star}$ are functions of $x$ and $y$, damping parameters, is called $a$ perfectly matched layer.

Let PML1, characterised by $\left(\sigma_{x_{1}}, \sigma_{x_{1}}^{\star}, \sigma_{y_{1}}, \sigma_{y_{1}}^{\star}\right)$ and PML2, characterised by $\left(\sigma_{x_{2}}, \sigma_{x_{2}}^{\star}, \sigma_{y_{2}}, \sigma_{y_{2}}^{\star}\right)$, be two perfectly matched layers. Suppose that $x=0$ is the interface between the two layers. If $\sigma=\sigma^{\star}$ and if $\sigma_{y 1}=\sigma_{y 2}$ then Bérenger has shown that the two layers PML1 and PML2 produce no reflection from the outgoing waves at the interface $x=0$. The damping parameter $\sigma$ is defined as $\sigma(\rho)=\sigma_{m}\left(\frac{\rho}{\delta}\right)^{2}$ where $\sigma_{m}$ is determined by the theoretical reflection at normal incidence, $\rho$ is the distance from the interface and $\delta$ is the thickness of the layer, see Bérenger [1]. We implement the perfectly matched layer method as follows: in the interior domain we used the evolution Galerkin scheme, i.e. by means of (2.6) in the case of EG-schemes or by (2.9), (2.10) in the case of finite volume EG-schemes. In the PML layer we used leap-frog scheme, see Bérenger [1] or Quan and Geers [18] for the discretisation of the PML layer. 


\section{Numerical Tests}

In the following numerical experiments we test and compare the absorbing boundary conditions using the methods of characteristics, extrapolation, the Laplace transformation and the perfectly matched layer (PML). The initial data are given either as plane waves or as pulses centered at certain points in the calculation domain. These numerical tests indicate that all the developed absorbing boundary conditions worked correctly in the case of one dimensional problems. However it is well known that there are big problems with waves having an incident angle not normal to the boundary. Therefore, for multidimensional problems the PML method gives the best results.

\section{Example 4.1}

To test the reflecting and the absorbing boundary conditions that are based on the method of characteristics we consider the wave equation system (3.1) and take $\Delta x=\Delta y=1, \Delta t=0.5$, $c=1$ and $\Omega=[0,100] \times[0,100]$. In Figure 2 the initial incident half sine wave which is taken from the wave $\phi_{0}=\sin \left(\frac{\pi x}{24}\right), u_{0}=\sin \left(\frac{\pi x}{24}\right)$ and $v_{0}=0$ is moved to the left and is reflected at the boundary $x=0$. In Figure 3 the Gaussian wave $\phi_{0}=0.5 \exp \left(-\ln (2)\left(\frac{x-10}{3}\right)^{2}\right), u_{0}=$ $-0.5 \exp \left(-\ln (2)\left(\frac{x-10}{3}\right)^{2}\right)$ and $v_{0}=0$ is moved to the right and is absorbed at the boundary $x=100$. We use the first order EG4 and the second order FVEG3 schemes, respectively. In Figure 2 we see that the one-dimensional half sine wave propagates to the left. Upon arrival at the left wall boundary it is reflected back. This happens after $\mathrm{T}=75.0$ (150 time steps). The phase of the reflected wave is opposite to that of the incident wave, as required by the reflected boundary condition. In Figure 3 the initial Gaussian wave propagates to the right. After the arrival at the right artificial boundary it leaves the domain as we see after $\mathrm{T}=62.5$ (125 time steps). We have made several other 1D examples for different initial data. All absorbing boundary conditions described above yield analogous results. These results indicate that such absorbing boundary conditions are doing well if the initial data are onedimensional data. Unfortunately, this is not the case if two-dimensional initial data are used as we will see in the Example 4.3.

\section{Example 4.2}

In this test we apply the PML method. We consider the two dimensional wave equation system inside the domain $\Omega=[-1,1] \times[-1,1]$. The initial data are taken to be

$$
\phi_{0}(x, y)=-\exp \left(-30\left((x+0.85)^{2}+y^{2}\right)\right), u_{0}(x, y)=0=v_{0}(x, y) .
$$

In the simulation we use a $100 \times 100$ mesh, the thickness of the matched layer is taken to be 10 cells and $\sigma_{m}$ is chosen such that the theoretical reflection at the normal incidence is $10^{-5}$. Inside the computational domain we update using the first order EG3 scheme. Inside the PML layer we use leap frog scheme. We take the Courant-Friedrichs-Lewy number CFL $=0.4$, we define it as $C F L=\frac{c \Delta t}{h}$, where $h=\Delta x=\Delta y$. We consider a time sequence, 0.2, 0.4, 0.6, 1.0, of absolute times $(\mathrm{T})$. The isolines of the solution $\phi$ are shown in Figure 4. Moreover, Figure 5 shows the result of the same problem using extrapolation for absolute times $\mathrm{T}=0.2$ and $\mathrm{T}=1.0$. Figure 5 clearly shows that, in general, using extrapolation as an absorbing boundary condition may produced unphysical reflected waves. It is clear from Figure 4 that the PML method produces only a marginal amount of reflection from the artificial boundaries. Thus in the case of the wave equation system this method is the preferable one in order to implement absorbing boundary conditions. 

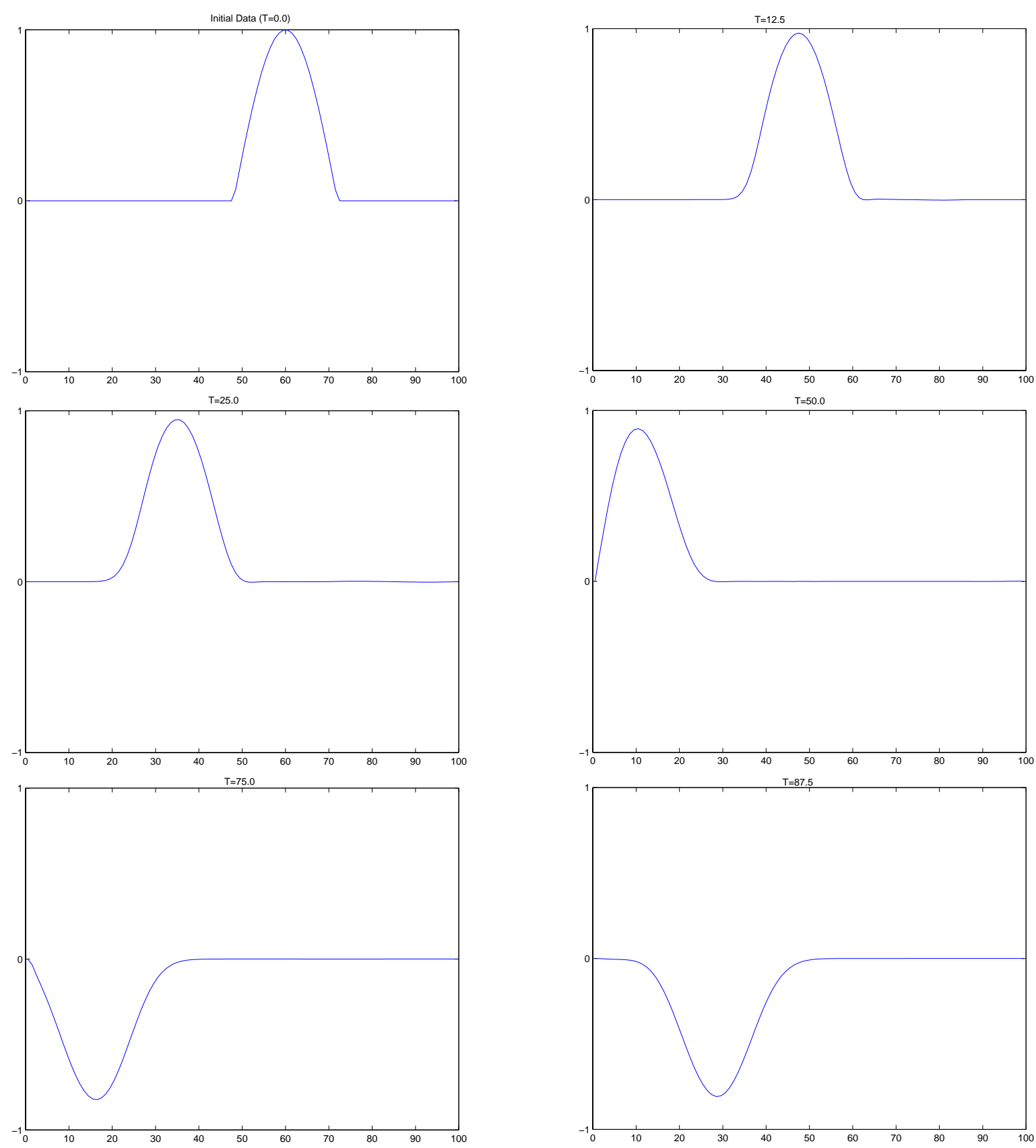

Figure 2: The numerical solution of the component $\phi$ using the EG4 scheme together with a reflector at $\mathrm{x}=0$. Half sine wave initial data. 

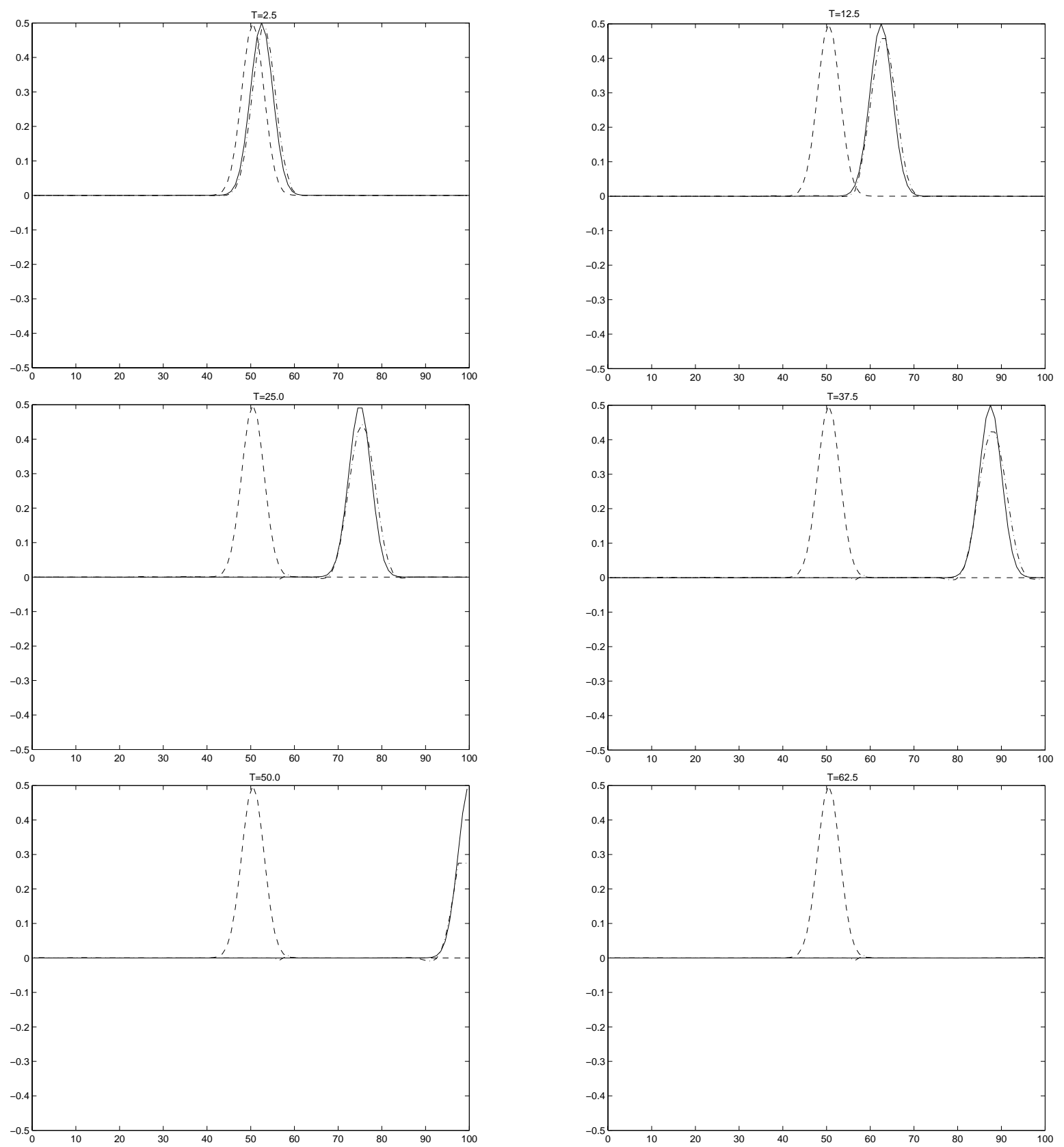

Figure 3: The initial data (- -), the exact solution (-) and the approximate solution (-.-.) of the component $\phi$ using the second order FVEG3 scheme. Absorber at $\mathrm{x}=100$. Gaussian puls initial data. 

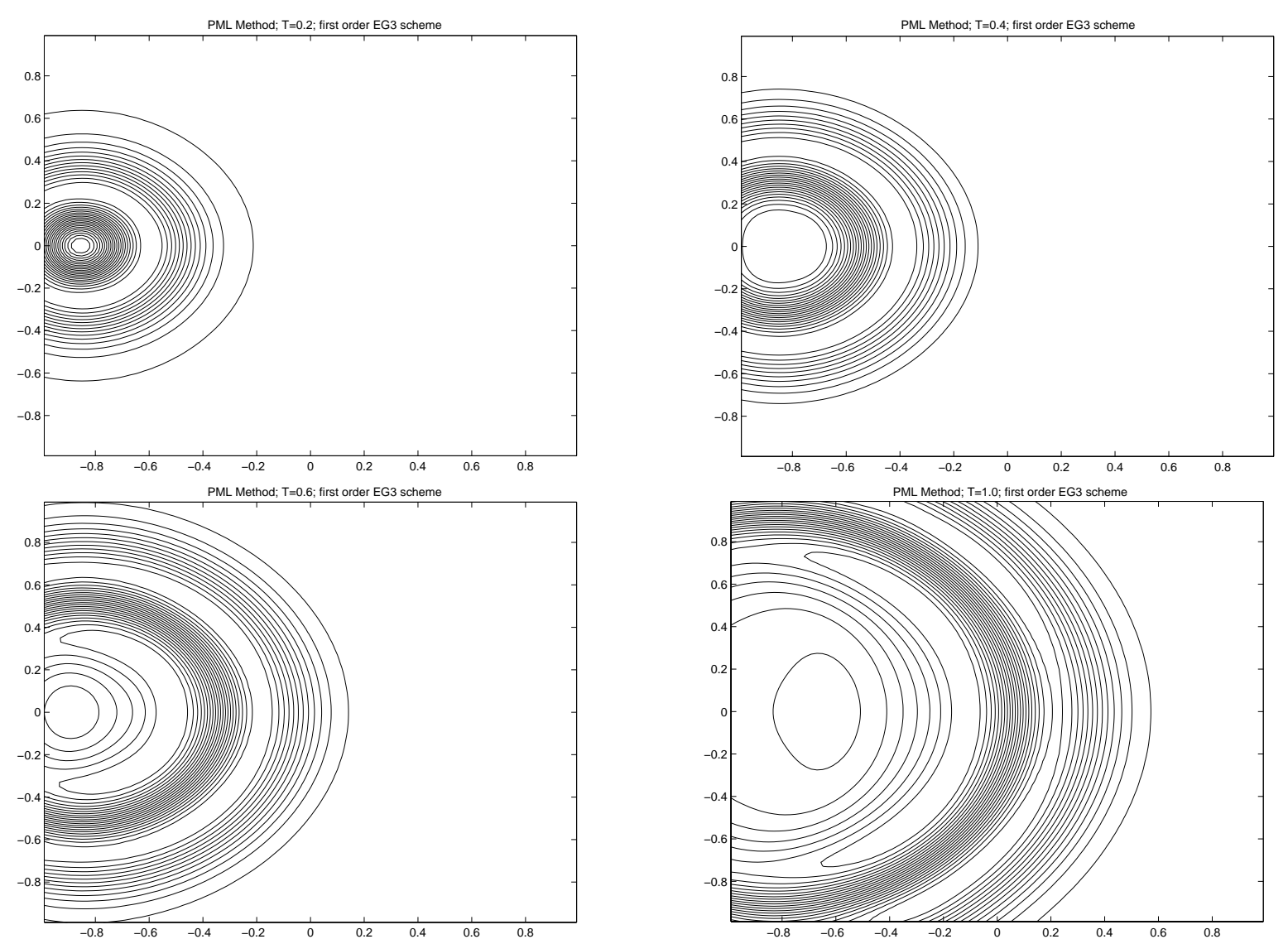

Figure 4: Isolines of the component $\phi$ of the approximate solution using the PML absorbing boundary condition.
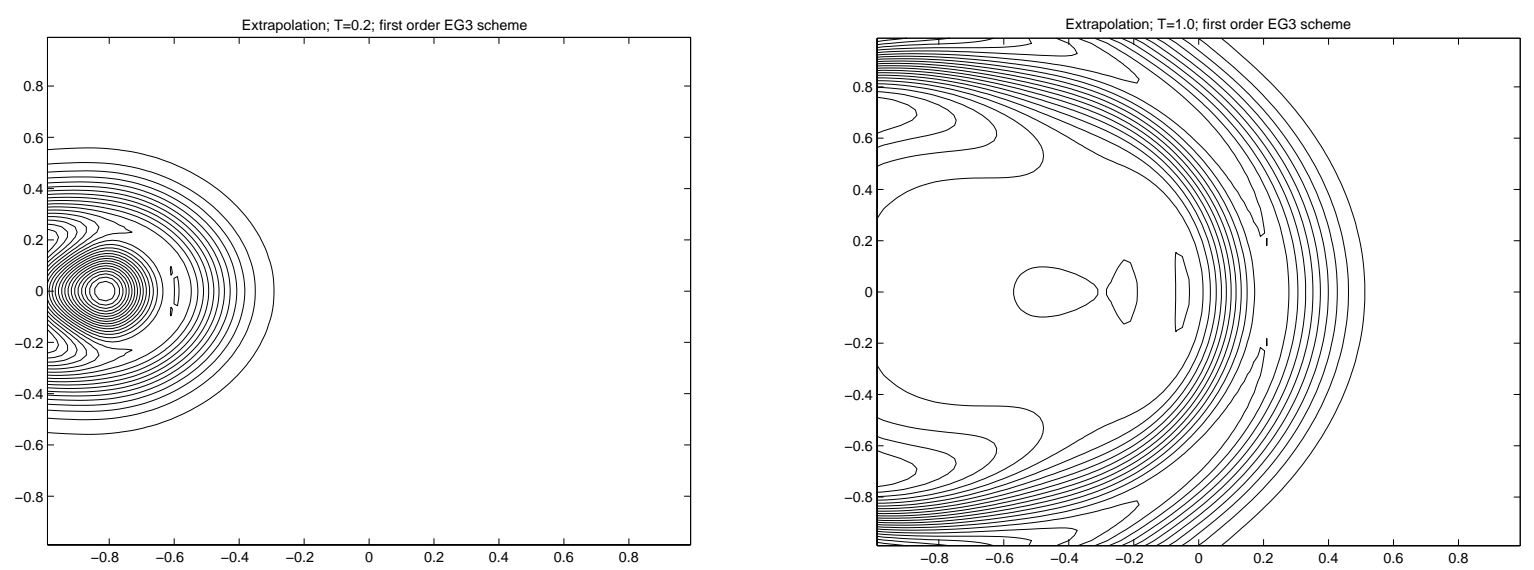

Figure 5: Isolines of the component $\phi$ of the approximate solution using extrapolation as an absorbing boundary condition. 


\section{Example 4.3}

In Example 4.1 we used absorbing boundary conditions that based on the methods of extrapolation and characteristics. The initial data were one-dimensional and such boundary conditions give good results with respect to preventing the outgoing wave from reentering the computational domain. However if the initial data are not one-dimensional then these boundary conditions produce reflected waves as we have already seen in Example 4.2. In this example we show that absorbing boundary conditions based on the method of characteristics and the Laplace transformation can also produce reflected waves in general. Again we consider the two-dimensional wave equation system inside the domain $\Omega=[-1,1] \times[-1,1]$. The initial data are taken to be

$$
\phi_{0}(x, y)=-\exp \left(-15\left((x+0.5)^{2}+y^{2}\right)\right), u_{0}(x, y)=0=v_{0}(x, y) .
$$

We use first and second order EG3 and FVEG3 methods and combine it with different boundary conditions. We take the $\mathrm{CFL}=0.55$, the absolute time $\mathrm{T}=0.825$ and consider $100 \times 100$ mesh. Figures 6 top-left and top-right show the isolines of the solution $\phi$ for the absorbing boundary condition using PML and extrapolation methods, respectively. Figures 6 bottomleft and bottom-right show the isolines using characteristics and Engquist-Majda first order condition methods, respectively. In Figure 7 we show the isolines of the solution $\phi$ for the reflecting boundary condition based on the method of characteristics at $x=-1$. These results demonstrate that the absorbing boundary conditions based on the method of characteristics as well as the one based on the Laplace transformation can produce much stronger unphysical oscillations and backward reflections. Pictures at the bottom of Figures 6 as well as picture at top-right are in fact comparable to the picture in Figure 7, where the result of the reflecting boundary condition is shown. In Figures 8 we use the second order FVEG3 method together with PML (top-left), extrapolation (top-right) and Engquist-Majda absorbing boundery condition of second order (bottom). The effect of the reflection is clear in top-right as well as in bottom pictures. Finally in Figure 9 we draw the isolines of $\phi$ for absolute times $\mathrm{T}=0.55$ and $\mathrm{T}=0.99$ when second order FVEG3 method is used together with extrapolation, PML and Engquist-Majda second order condition methods, respectively. We see that less effect of the boundary in the case of PML method.

\section{Conclusions}

In this paper we derive some absorbing and reflecting boundary conditions for the twodimensional wave equation system. These conditions are based on the known methods of characteristics, extrapolation, Laplace transformation (Engquist-Majda conditions) and PML. We use them with EG-schemes. Inside the computational domain we always used the EGschemes which are given in Section 2. The numerical experiments which we presented in this paper showed clearly that for one-dimensional cases all methods give good results. Further, for two-dimensional problems we noticed that the PML method produced less nonphysical oscillation and reflected waves when used together with EG-methods. In order to compare computational costs of different $\mathrm{ABC}$ techniques applying to EG-methods, we have taken one particular EG-method, e.g. the second order FVEG3. We carry out the calculations in Example 8 with meshes 100, 200 and 400, respectively. The results are tabulated below in Table 1. We see that the cheapest method is the method of extrapolation while the expensive one is clearly the PML method. 

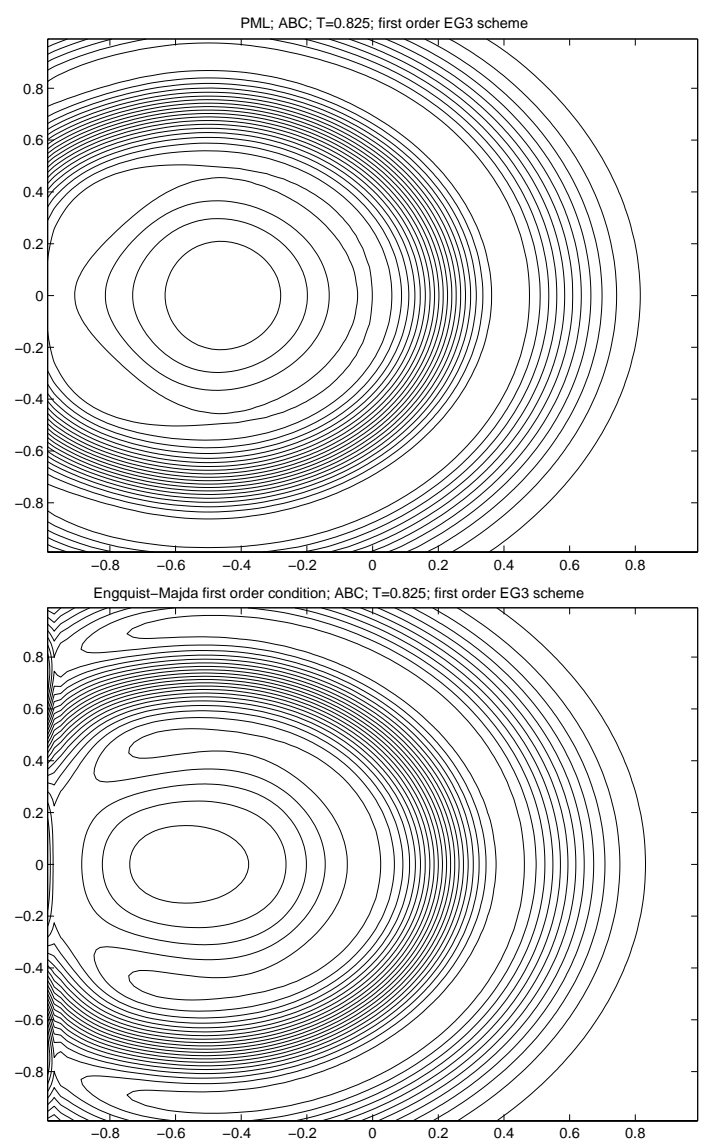
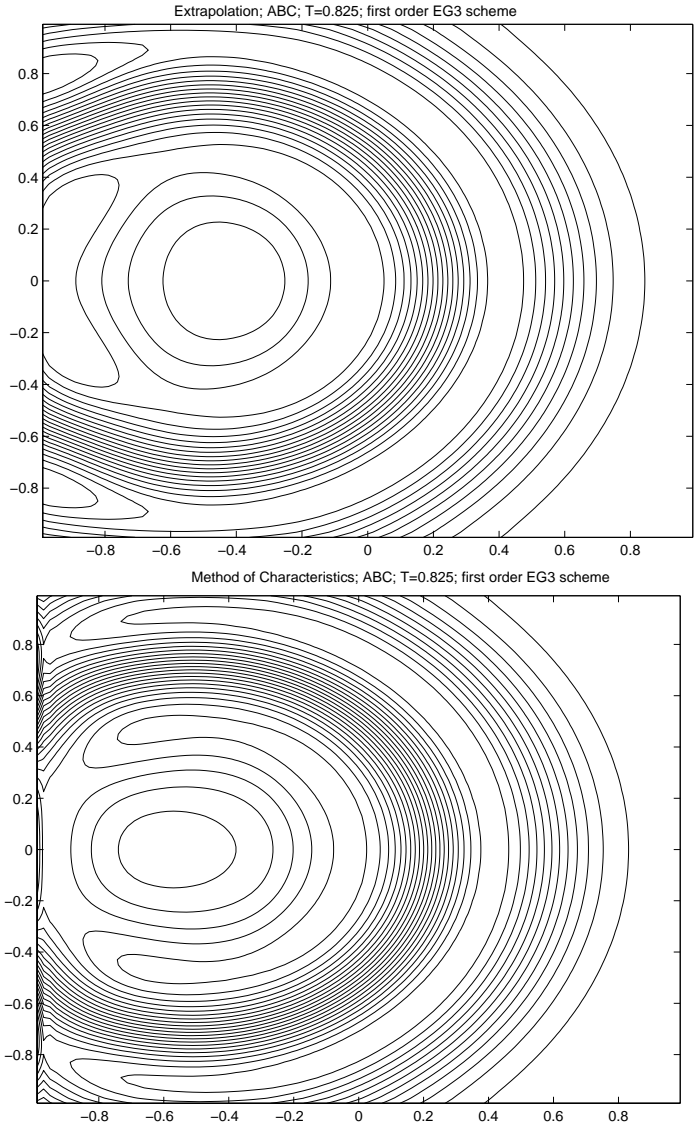

Figure 6: Isolines of the component $\phi$ of the approximate solution, absorber based at $x=-1$ : top-left: based on the PML, top-right: based on extrapolation, bottom-left: based on the method of characteristics and bottom-right: based on Laplace transform.

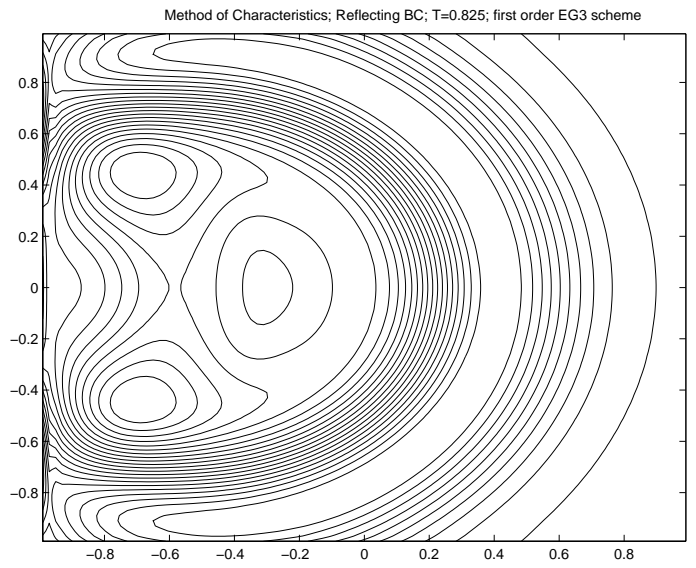

Figure 7: Isolines of the component $\phi$ of the approximate solution, reflector based on the method of characteristics at $x=-1$. 

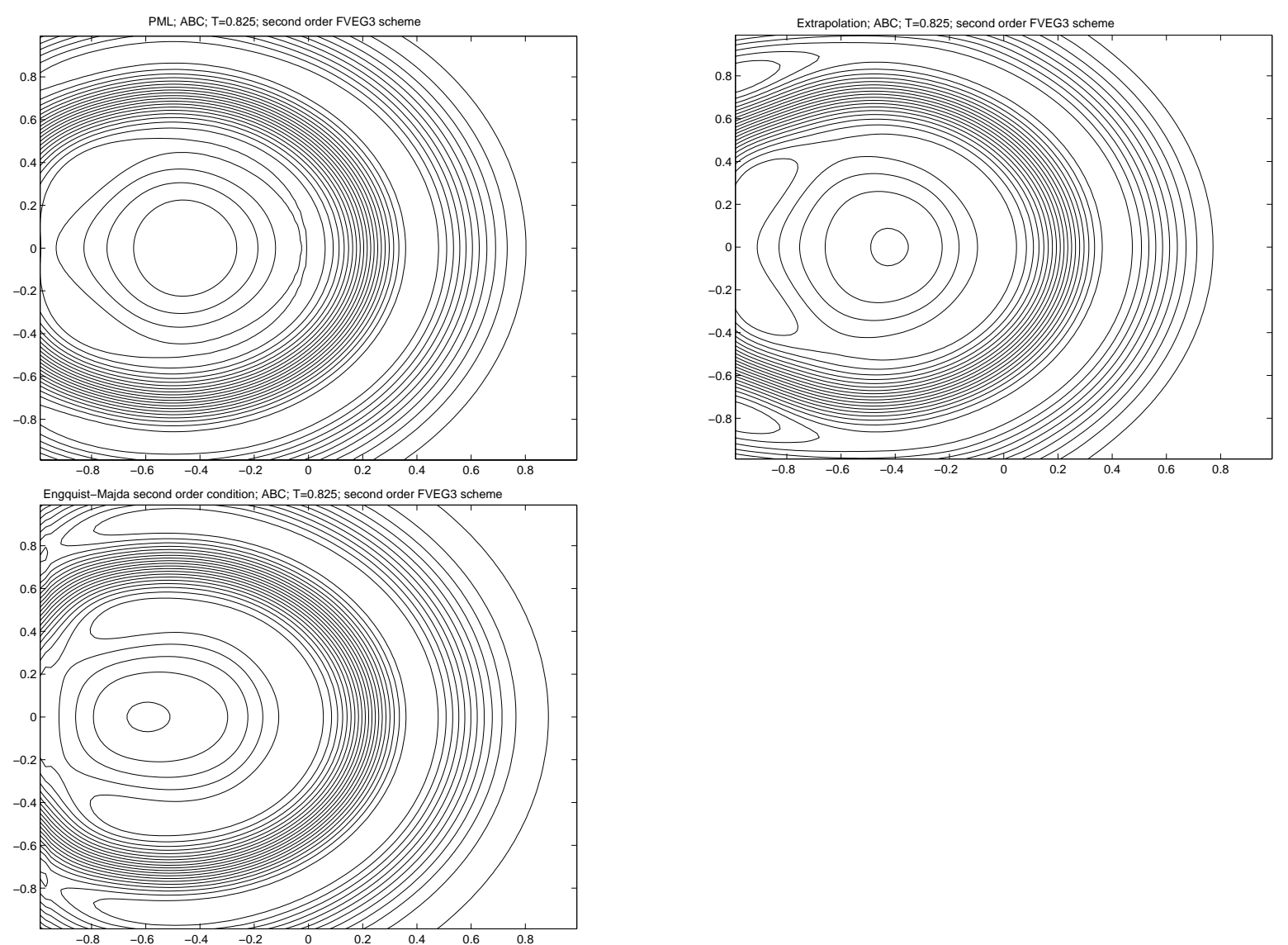

Figure 8: Isolines of the component $\phi$ of the approximate solution, absorber based on the PML (top-left), on extrapolation (top-right) and on Engquist-Majda second order condition (bottom). 

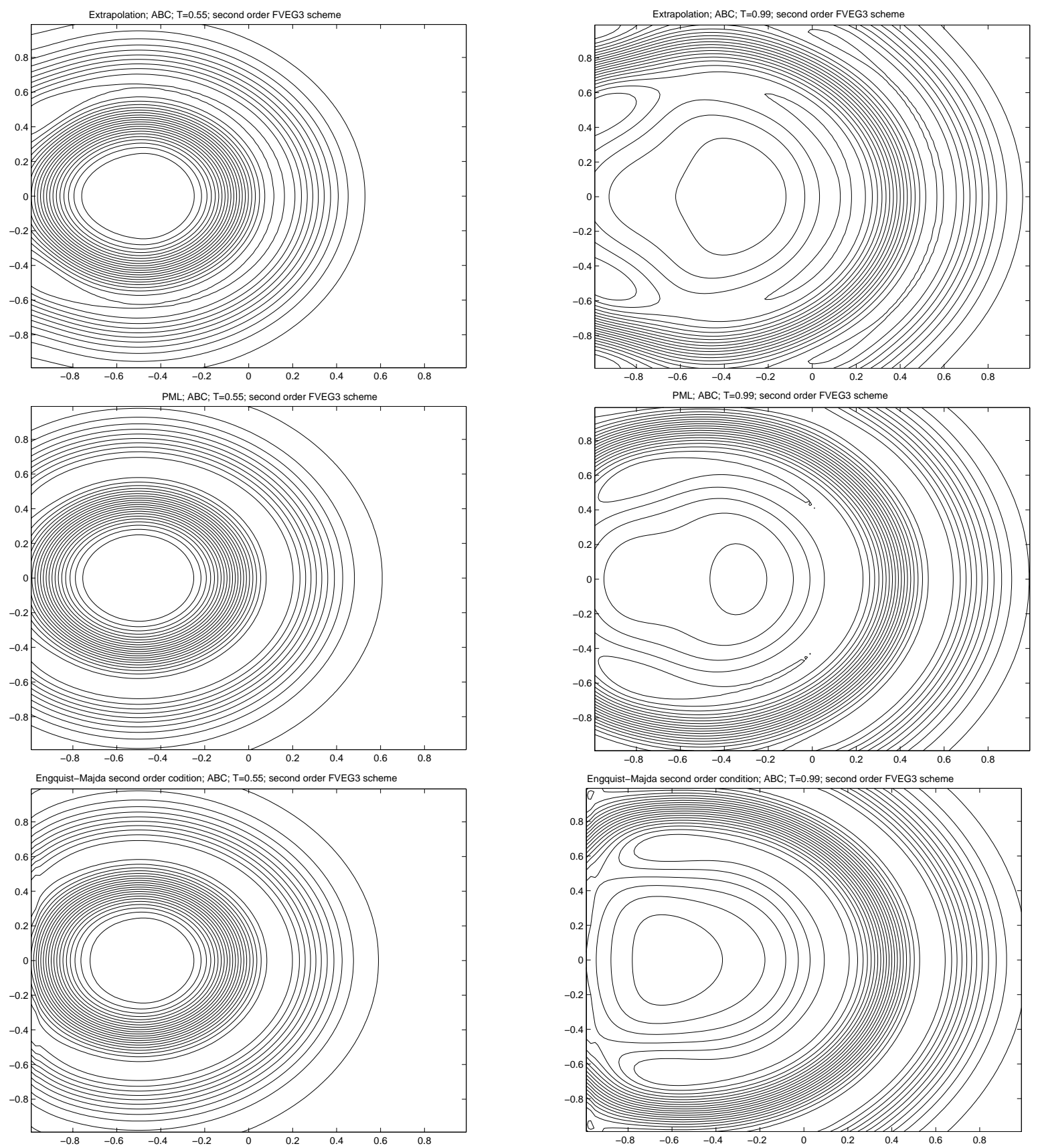

Figure 9: Isolines of the component $\phi$ of the approximate solution using extrapolation, PML and Engquist-Majda second order condition methods. 


\begin{tabular}{|c|c|c|c|c|}
\hline & Method & PML & Extrapolation & Engquist-Majda second order condition \\
\hline $100 \times 100$ & cost & $0.92 \mathrm{~s}$ & $0.01 \mathrm{~s}$ & $0.43 \mathrm{~s}$ \\
$200 \times 200$ & cost & $6.00 \mathrm{~s}$ & $0.06 \mathrm{~s}$ & $3.26 \mathrm{~s}$ \\
$400 \times 400$ & cost & $44.84 \mathrm{~s}$ & $0.31 \mathrm{~s}$ & $25.82 \mathrm{~s}$ \\
\hline
\end{tabular}

Table 1: Computational time results.

With this paper we have demonstrated that it is feasable to implement boundary conditions within the framework of evolution Galerkin schemes based on bicharacteristics.

\section{Acknowledgements}

This research was supported under the DFG grant No. Wa 633/6-2 of Deutsche Forschungsgemeinschaft, by the grants GACR 201/00/0557 and CZ 39001/2201 of the University of Technology Brno as well as by the VolkswagenStiftung and DAAD Agencies. We would like to thank the referees for a large number of helpful comments which enabled us to improve the paper.

\section{References}

[1] J.-P. Bérenger. A perfectly matched layer for the absorbtion of electromagnetic waves. J. Comput. Phys., 114:185-200, 1994.

[2] A. Bradly, L.Greengard, and T. Hagstrom. Nonreflecting boundary conditions for the time-dependent wave equation. J. Comput. Phys., 180(1):270-296, 2000.

[3] B. Engquist and A. Majda. Absorbing boundary conditions for the numerical simulation of waves. Math. Comput., 31(139):629-651, 1977.

[4] M. J. Grote. Nonreflecting boundary conditions for time dependent wave propagation. Seminar für Angewandte Mathematik (SAM), Research report No. 2000-04, Zürich, 2000.

[5] M. J. Grote and J. B. Keller. Exact nonreflecting boundary conditions for the time dependent wave equation. SIAM Journal of Appl. Math., 55(2):280-297, 1995.

[6] M. J. Grote and J. B. Keller. Nonreflecting boundary conditions for time-dependent scattering. J. Comput. Phys., 127:52-65, 1996.

[7] M. J. Grote and J. B. Keller. Nonreflecting boundary conditions for Maxwell's equations. J. Comput. Phys., 139:327-342, 1998.

[8] G. W. Hedstrom. Nonreflecting boundary conditions for nonlinear hyperbolic sytems. $J$. Comput. Phys., 30:222-237, 1979.

[9] R. L. Higdon. Numerical absorbing boundary conditions for the wave equation. Math. Comput., 49(179):60-90, 1987.

[10] R. Holland and J. W. Williams. Total-field versus scattered-field finite-difference codes: A comparative assessment. IEEE Transaction on Nuclear Science, NS-30(6):4583-4588, 1983. 
[11] Fang Q. Hu. On absorbing boundary conditions for linearized Euler equations by a perfectly matched layer. J. Comput. Phys., 129:201-219, 1996.

[12] M. Lukáčová, K. W. Morton, and G. Warnecke. Finite volume evolution Galerkin methods for Euler equations of gas dynamics. Int. J. Num. Methods in Fluids, accepted, 2001.

[13] M. Lukáčová, K.W. Morton, and G. Warnecke. Evolution Galerkin methods for hyperbolic systems in two space dimensions. Math. Comput., 69(232):1355-1384, 2000.

[14] M. Lukáčová, J. Saibertová, G. Warnecke, and Y. Zahaykah. On evolution Galerkin methods for the Maxwell and the linearized Euler equations. Accepted to (Appl. Math.), 2003.

[15] M. Lukáčová, G. Warnecke, and Y. Zahaykah. Third order finite volume evolution Galerkin (FVEG) methods for two-dimensional wave equation system. submitted to East-West J. Numer. Math., 2002.

[16] G. Mur. Absorbing boundary conditions for finite-difference approximation of the timedomain electromagnetic field equations. IEEE Transaction on Electromagnetic Compatability, EMC-23(4):377-382, 1981.

[17] S. Ostkamp. Multidimensional characteristic Galerkin schemes and evolution operators for hyperbolic systems. Math. Meth. Appl. Sci., 20:1111-1125, 1997.

[18] Quan Qi and T. L. Geers. Evolution of the perfectly matched layer for computational acoustics. J. Comput. Phys., 139:166-183, 1998.

[19] K. W. Thompson. Time dependent boundary conditions for hyperbolic systems. J. Comput. Phys., 68:1-24, 1987.

[20] K. W. Thompson. Time dependent boundary conditions for hyperbolic systems, II. J. Comput. Phys., 89:439-461, 1990. 